\title{
Relación entre algunos milestones tecnológicos y la evolución de los registradores industriales
}

\author{
L.R. Vega-González \\ Centro de Ciencias Aplicadas y Desarrollo Tecnológico, CCADET \\ E-mail:lrvg@servidor.unam.mx
}

(Recibido: noviembre de 2005; aceptado: septiembre de 2006)

\section{Resumen}

Los mile stones son las "piedras de señalamiento de milla" que encontramos en las carreteras norteamericanas, las cuales nos indican cuánto camino hemos recorrido. La palabra milestone también significa un punto significante en el desarrollo, (Merriam Webster, 1996). Al estudiar la evolución tecnológica de la instrumentación industrial, se observa lo que parece ser una alta correlación entre la aparición de nuevos instrumentos y la disponibilidad y aplicación de algunos dispositivos o tecnologías sobresalientes, o mile stones tecnológicos. A lo largo de los años, estos dispositivos han permitido implementar algunos subsistemas para resolver diversos problemas y tareas no resueltas en los instrumentos, según el momento evolutivo por el que pasaba la indu stria. Esta situación resulta muy evidente al estudiar el caso de los registradores electrónicos industriales, ya que como se muestra en este trabajo, diferentes dispositivos tecnológicos ayudaron a resolver los problemas técnicos y contribuyeron con diversas aportaciones que mejoraron la operabilidad de los instrumentos en sus épocas de vigencia. Así pues, se puede decir que detrás de la aparición de cada nuevo instrumento o familia de instrumentos, se encuentra al menos un dispositivo tecnológico sobresaliente o mile stone. En este artículo trataremos de mostrar evidencias sobre la hipótesis ante rior, al examinar el caso de una línea de registradores electrónicos industriales.

Descriptores: Registradores industriales, evolución tecnológica, mile stones tecnológicos.

\begin{abstract}
Mile stone means a post serv ing as a mile post sig nal and also a sig nif i cant point in de velop ment, Merriam Webster (1996). In the industrial recorderstechnologicalevolu tionary stud ies, it is ob served that when new in stru ments were launched to mar ket there were avail able some out stand ing new tech nologydevices, or tech nolog ical milestones. Through the years, those devices had per mit ted to im ple ment in stru ment subsys tems that over come previ ously non solved prob lems. This is ev i dent when we study in dus trial electronic re cord ers, be cause, as shown in this work, many differ ent tech no log ical de vices helped to solve tech nical prob lems im prov ing in stru ment operability in different time pe riods. Then, it is pos sible to say that be halfeach new in stru ment or in stru ment fam ily, there are al least an out stand ing tech no log ical de vice or mile stone. In this ar ticle we will show some ev idence about the men tioned hy pothe sis, study ing the case of in dus trial elec tronicrecorders.
\end{abstract}




\section{Los milestones tecnológicos}

El término milestone es utilizado en distintas disciplinas, por ejemplo, en la administración de proyectos; los milestones son los puntos de control en el tiempo que señalan eventos importantes en los programas de ruta crítica, [1]; en pediatría, los milestones son las distintas etapas en el desarrollo del habla de los niños, [4]. En todos los casos, esta palabra se refiere a hechos o acontecimientos importantes.

Así, cuando hablamos de milestones tecnológicos, podemos referirnos a grandes sucesos en la historia de la tecnología mundial integrada, por ejemplo, la conclusión y puesta en operación de la Planta Hidroeléctrica Adams el 26 de Agosto de 1895 en las Cataratas del Niagara, en Búfalo New York, EUA, que representó la victoria de los sistemas de corriente alterna, sobre los sistemas de corriente directa; o bien, la conclusión de la Computadora e Integrador Numérico Electrónico ENIAC en 1946, en la Universidad de Pennsylvania, Filadelfia EUA, hecho que impulsó el desarrollo de las computadoras modernas de propósito general, IEEE [3]. Por otra parte, cuando se citan aspectos sobresalientes de alguna tecnología con menor grado de integración, también se habla de milestones. Tal es el caso de los paneles solares [5] o la cromatografía de gases [2], entre otros muchos ejemplos.

En el presente trabajo, nos referiremos como milestones tecnológicos a aquellos dispositivos que constituyeron innovaciones de ruptura al integrarse en los registradores industriales. Por la evidencia empírica podemos afirmar, que en el desarrollo de todas las categorías de instrumentos industriales, tales como controladores, analizadores, válvulas y sistemas de medición de todo tipo, han existido milestones tecnológicos de gran importancia, los cuales han posibilitado las mejoras tecnológicas integrales de los equipos. A continuación, se presentan los milestones que cimentaron el camino de la industria de los registradores industriales.

\section{Instrumentación para registro}

Un registrador es un dispositivo tecnológico que proporciona una imagen gráfica del comportamiento de alguna variable dinámica de proceso, tal como la presión, el nivel, la temperatura, la potencia eléctrica o la conductividad electrolítica, entre muchas otras.

Es un instrumento muy importante para los operadores de las plantas de proceso industriales y para los analistas químicos. Puede medir, indicar, registrar e incluso controlar un proceso desatendido por varios días.

Los mecanismos que posibilitaron la implementación de los primeros registradores aparecieron durante el siglo XVII. El sistema activo del sistema mecánico podía ser un diafragma, fuelles o un tubo helicoidal, el cual era energizado por aire, fluidos o movimientos mecánicos. La gráfica era del tipo rollo o circular.

En la mayoría de los casos, la medición de las variables la realizaba el sistema mecánico activo y la salida del instrumento eran los registros producidos por el trazo de tinta sobre el papel.

Para principios del siglo XVIII ya existían los instrumentos del tipo de "escritura directa", en los cuales, la pluma se sujetaba al sistema activo del registrador.

La figura 1 muestra un ejemplo de registrador de escritura directa en el que se tuvo que diseñar y utilizar un sistema de impulso con la sensibilidad suficiente para reducir la fricción de la pluma y lograr registros continuos.

En los sistemas eléctricos el elemento activo fue el galvanómetro de deflexión de alta sensibilidad, el cual respondía a señales eléctricas. Este dispositivo que se muestra en la figura 2 apareció a finales del Siglo XVIII.

El galvarómetro de deflexión puede ser considerado como la primer innovación tecnológica 


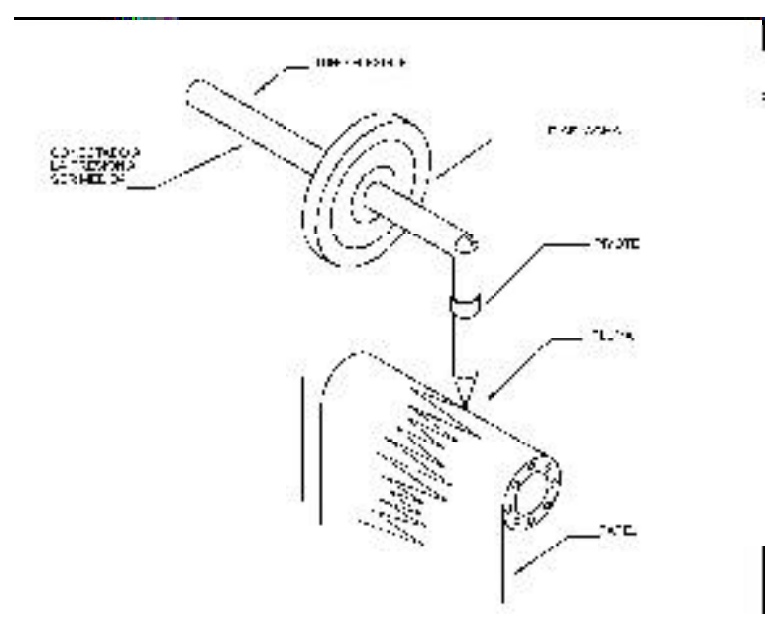

Figura 1. Registradores de escritura directa

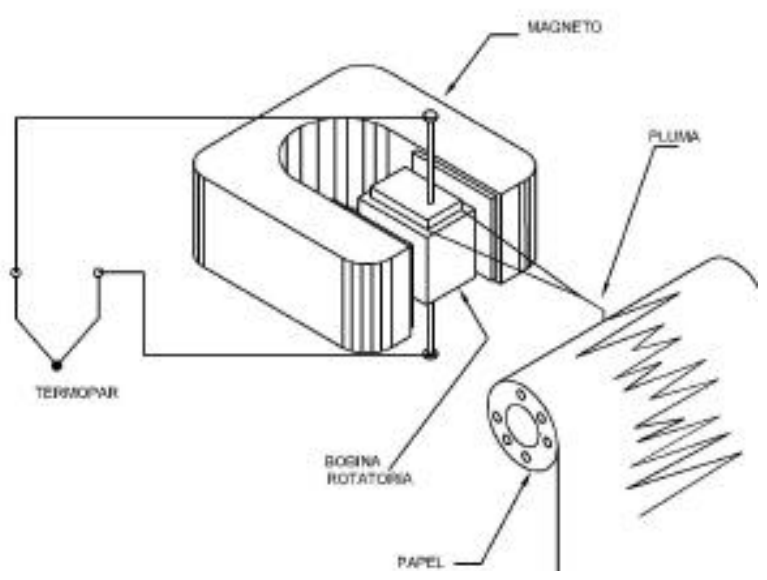

Figura 2. Registrador eléctrico por galvanómetro de deflexión (1890).

de ruptura o milestone tecnológico, ya que combinado con el uso de los circuitos de medición potenciométrico y el puente de Wheatstone, trabajando en corriente directa, permitieron el desarrollo del registrador electromecánico llamado "Callendar". Este equipo ha sido reconocido en la literatura como el primer registrador con la característica de balance continuo.

El desbalance producido por la señal que se medía en un circuito puente o potenciométrico, se detectaba por un galvanómetro, el cual actuaba a travé s de relevadores del circuito de balance. Este sistema de detección resultó muy delicado, por lo cual no fue satisfactorio para uso industrial.

Un par de décadas más tarde, hacia el año 1913, la compañía Leeds \& Northrup desarrolló un registrador de uso general de tipo electromecánico que requería 30 segundos para balancear en una escala total de 10 pulgadas, (Clark, 1966). Este instrumento se fabricó hasta 1956, utilizaba un galvanómetro de alta sensibilidad para detectar el grado de desbalance en el circuito de medición. El registro del punto medido se realizaba con ayuda de un amplificador mecánico que proveía el par necesario para rebalancear el circuito y mover la pluma registradora al punto de la nueva medición en la escala.

Cuando el circuito de medición estaba desbalanceado, el galvanómetro se llevaba a una posición de "fuera de cero". Los sensores del motor detectaban dicha posición y provocaban que la resistencia deslizable "slidewire" del circuito de medición se moviera hasta la nueva posición de balance. 
La resistencia deslizable o slidewire puede considerarse como un nuevo milestone tecnológico, ya que este dispositivo posibilitó el cierre de la malla de realimentación del registrador de balance nulo, para obtener un registrador de balance eléctrico continuo. En cada ciclo de operación, el galvanómetro actuaba con movimiento libre y el resto del tiempo regresaba a la posición original con propósito de rebalanceo, esto es, regresaba a cero entre medición y medición, por lo que a este instrumento se le conoció como registrador "paso por paso".

El puntero de la escala, indicaba el valor desconocido que se medía y una pluma registraba continuamente la medición en el papel.

El amplificador mecánico de este instrumento producía el par suficiente para manejar la pluma registradora, las señales de alarma, los contactos, la resistencia de control (slidewire), así como los motores sensitivos, todo lo cual actuaba por estar montado a la flecha de la resistencia de control.

En sus últimas versiones, estos instrumentos podían registrar entradas múltiples, ya que contaban con selectores e interruptores accionados mecánicamente por el movimiento del motor. Los circuitos de medición estaban diseñados para que la corriente en el detector se hiciera cero para todas las posiciones de balance, por lo cual también fueron llamados "registradores de balance nulo", con amplificador mecánico.

A principios de los años 50's del siglo pasado, los registradores de balance nulo eléctrico con amplificador mecánico, mejoraron sus especificaciones sustancialmente. Principalmente, se logró que el balance a escala total tomara 12 segundos como mínimo, aunque la medición estaba limitada exclusivamente a señales de termopar con un rango mínimo de 8 milivolts. Estos equipos presentaban fallas cuando su operación era expuesta a vibraciones.

\section{El primer registrador con amplificador electrónico}

En 1932, aún dentro del período de madurez de la tecnología del registrador de balance nulo mecánico, Williams, de la empresa Leeds \& Northrup, inventó el registrador con amplificador electrónico, el cual fue difundido mundialmente bajo la marca registrada Speedomax. ${ }^{\circledR}$, (Leeds and Northrup Co. 1971,1972).

Su desarrollo fue posible, ya que se utilizaron los siguientes milestones o innovaciones tecnológicas de ruptura: el amplificador básico con válvulas de vacío tipo tiratrón y el Chopper tipo micrófono. Además, se mejoró la fabricación del slidewire de realimentación por medio del uso de nuevas aleaciones metálicas que mejoraron la respuesta del circuito de medición del instrumento, como se explica a continuación.

En la figura 3 puede verse que estos instrumentos usaban un circuito de medición tipo potenciométrico, en el cual el desbalance de corriente directa se convertía a corriente alterna por medio del circuito "chopper", permitiendo así su amplificación por medio de válvulas de vacío y usando el voltaje resultante para encender los tiratrones rectificadores.

Cuando el sensor al medir provocaba un desbalanceo, el tiratrón respectivo, según el signo de la señal, encendía, energizando al motor. Este último llevaba la resistencia deslizable a la nueva posición de balance.

Los primeros sistemas tipo chopper, estaban basados en resortes mecánicos, vibradores y micrófonos de carbón; sin embargo, la vida útil del instrumento era limitada debido a la falta de materiales de larga vida y duración.

La función del chopper era convertir la corriente directa en corriente alterna para lograr un funcionamiento más apropiado del amplificador. Uno de los inconvenientes de esta tecnologí a, era que cuando el chopper se exponía a vibraciones provocaba fallas. 


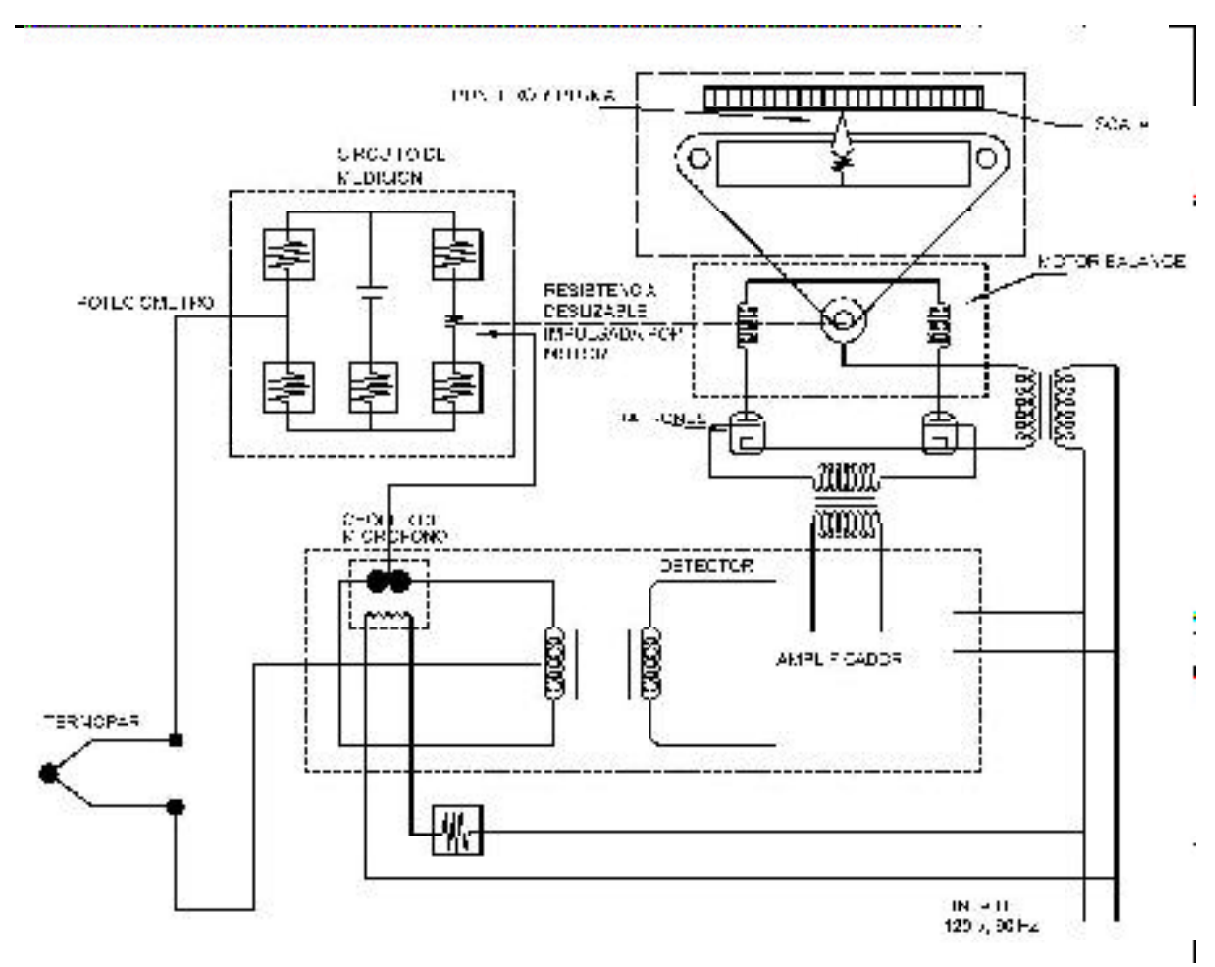

Figura 3. Diagrama de bloques de un registrador con amplificador de válvulas de vacío y chopper

En este tipo de equipos se mejoraron las especificaciones del voltaje de entrada, ya que se podía medir y detectar un voltaje mínimode $2 \mathrm{mV}$, con un error de $+/ 0.255 \%$ y con velocidad de balance a escala total de 1 segundo, aproximadamente.

Este rango posibilitó que el uso de los registradores ya no estuviera sólo limitado para la entrada de termopares, permitiendo realizar la medición de otros transductores y sensores eléctricos.

Entre la década de 1930 a 1940, diferentes compañías lanzaron al mercado algunas otras versiones de registradores electrónicos. Esterline Angus desarrolló el primer registrador en base a servomotor, obteniendo una respuesta de $1 / 8$ de segundo escala total. La bobina del servomotor incluía una bobina impulsora y una bobina de velocidad, soportada en baleros y trabajando bajo el campo magnético de un imán permanente.
Por otra parte, Bailey Meter Company produjo y patentó el Galvatrón, mientras que Tagliablue Manufacturing Co., fabricó otro registrador de balance nulo, con la variante de que usaba un haz de luz en la medición y un galvanó metro tipo espejo, un fototubo, un amplificador, un relevador y un motor.

\section{Registradores de balance nulo 1960-1970}

Los registradores con amplificador de válvulas de vacío, permanecieron en el mercado hasta finales de la década de los cincuentas. El nuevo avance en los equipos de registro industriales se dio hasta mediados de la siguiente década, basados en el uso de las siguientes tecnologías relevantes: un amplificador transistorizado y un servosistema de alta precisión constituido por un motor de balance de dos fases reversible y un nuevo milestone, el convertidor electromecánico sincrónico. Estos instrumentos fueron registrados con la marca comercial de Speedomax H\&W $®$, (Leeds and Northrup Co. 1972,1975). 
La nueva tecnología mejoró las especificaciones de estos instrumentos logrando un rango de entrada mínimo de $1 \mathrm{mV} \mathrm{CD}$, con un tiempo de balance de entre 2 a 4 segundos por punto. El sistema de entintado cambió de tinteros de vidrio o plástico alimentados por capilar a las plumillas, al sistema de cartuchos de tinta. También se mejoró el sistema de alimentación de papel por las gráficas de rollo.

Otro aspecto relevante de estos registradores fue que la tecnología de transistores permitió la inclusión de un circuito de compensación por junta de referencia fría para las mediciones de temperatura por termopar.

Esto resolvió el problema de error parásito que se genera en las uniones de las terminales del termopar con el cable de extensión. El cable de extensión se usa desde el punto de medición en donde está montado el termopar, hasta el cuarto de control o gabinete en donde está montado el registrador. En la unión entre el termopar y el cable de extensión, se forma una nueva junta de termopares parásitos, también conocida como junta fría. Ésta provoca la aparición de un milivoltaje de error en la medición (Figura 4).
En los instrumentos con tecnología mecánica y aún de bulbos, este efecto se corregía compensando mecánicamente la posición del puntero indicador y dibujando la escala en forma no lineal. Los amplificadores transistorizados permitieron corregir el offset de manera electrónica, por lo que todas las escalas de indicación se pudieron dibujar linealmente en lo sucesivo.

La rapidez de balance de estos equipos, debido a su servosistema optimizado, dio origen al nuеvo concepto de registrador multipunto. Los instrumentos multipunto de primera generació $n$ podían ahora medir y registrar hasta 30 puntos en una rutina de medición de 1 minuto. Para eliminar los conflictos entre medición y medición, se tuvo que diseñar una lógica de arbitraje, la cual fue realizada por medio de lógica de relevadores combinacional.

\section{Registradores de una, dos o tres plumas 1970-1980}

Una nueva generación de registradores transistorizados apareció durante la década de los años setenta del siglo pasado y fue registrada bajo la marca Speedomax 165\&250 ®, (Leeds and Northrup Co. 1979,1980).

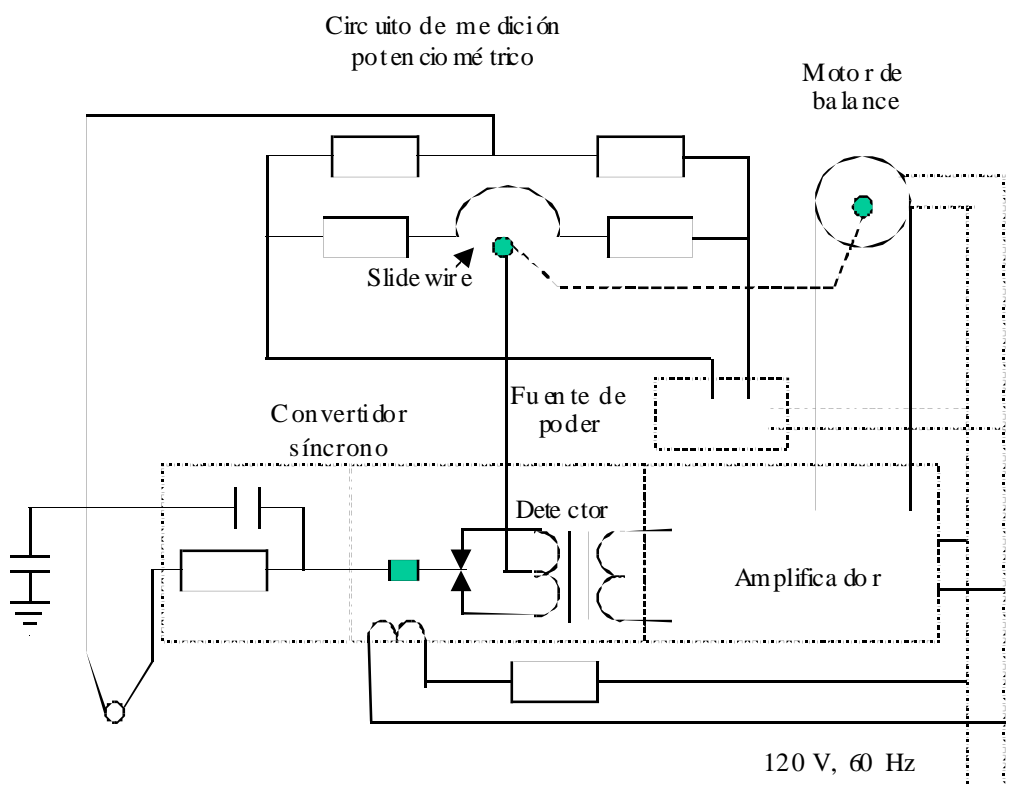

Figura 4. Diagrama de bloques de un registrador de balance nulo transistorizado 


\section{L.R. Vega-González}

También se incorporaron a las tarjetas madre de estos instrumentos, los circuitos digitales del tipo CMOS con Tecnología ULSI, los cuales agregaron una serie de características que los hizo más atractivos, versátiles, adecuados y adaptables a cualquier necesidad de registro. La lógica permitió la selección e identificación clara de puntos en la impresión y la capacidad multirango a través del multiplexaje sincrónico de entradas y el manejo de distintos arreglos de alarmas.

En la figura 5 pueden observarse los módulos o tarjetas que identifican las características tecnológicas sobresalientes en estos instrumentos, tales como el indicador con despliegue digital del punto medido y la impresión en papel termo sensible. Cabe aclarar que se conservó la opción de impresión a tinta.
En este instrumento, las entradas del usuario se conectaban a una tarjeta de rango con ajustes de cero y gama, la cual a su vez, alimentaba a un preamplificad or para llevar la señal de bajo nivel, hasta 1.5 Volts.

En la tarjeta madre "A" se realiza la interconexión y control central de todas las partes del sistema, motor de balance, resistencia deslizable, transformador de potencia, etcétera.

De forma similar a los sistemas usados en otros registradores, la salida del servoamplificador impulsa al motor de balance, el cual se mueve para corregir el error, moviendo también la posición de la resistencia deslizable, cuyo seguidor se realimenta al servoamplificador.

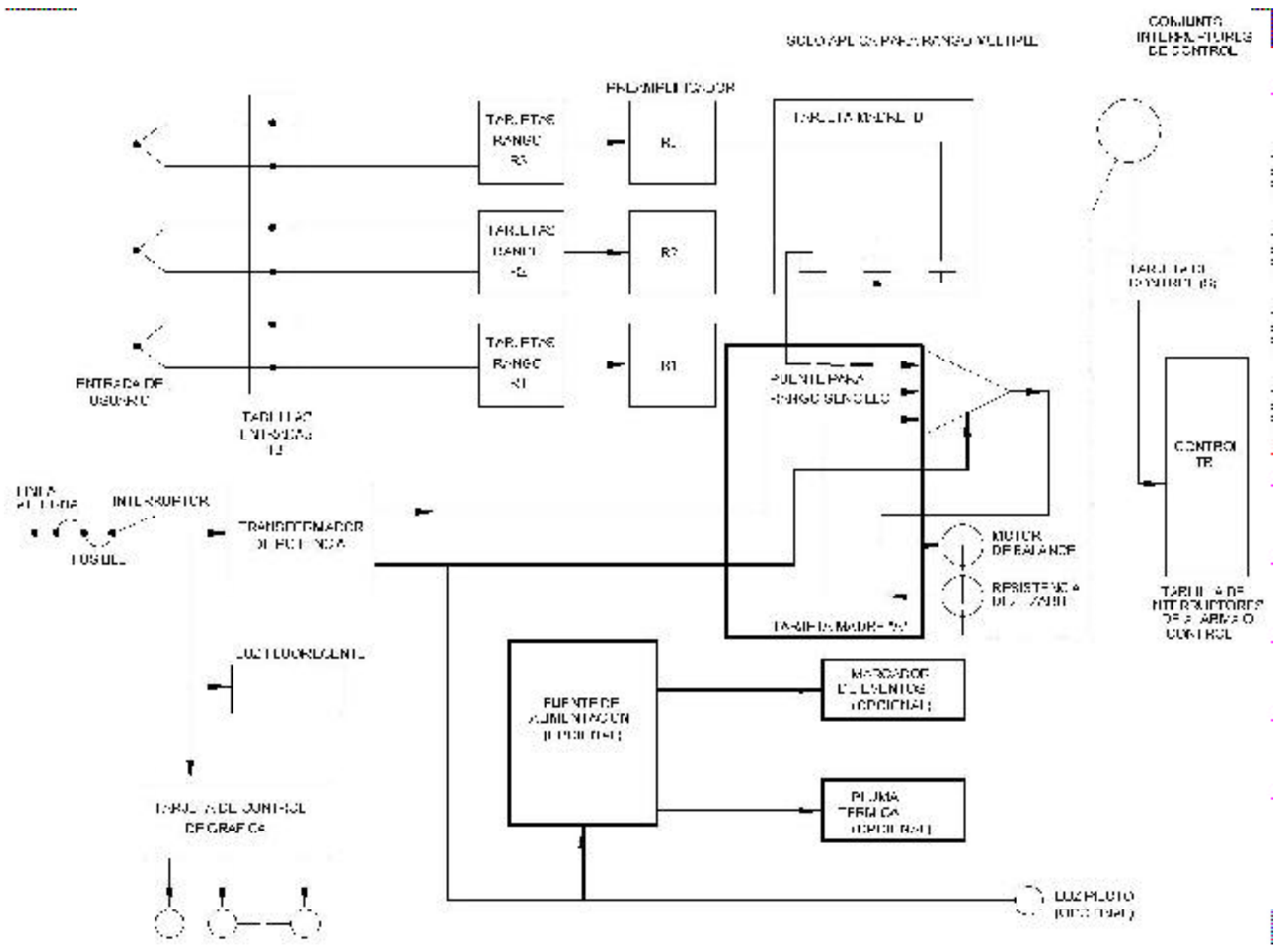

Figura 5. Diagrama de bloques de un registrador digital de una, dos o tres plumas 
Al igual que todos los otros dispositivos que constituyen el registrador, la cabeza impresora actúa en forma sincrónica bajo el comando de la tarjeta de lógica.

Registradores Multipunto Digitales de $2^{\mathrm{a}}$ generación, Speedomax 165 \& 250 L\&N $®$. (Leeds and Northrup Co. 1979,1980).

La versión multipunto de los registradores Speedomax 165\&250, con hasta 30 puntos de registro, fue muy usada en la práctica. Su diagrama de bloques se muestra en la figura 6 .

La variante principal respecto a los instrumentos de una, dos o tres plumas, es que la lógica combinacional realizada en la Tarjeta Madre "A", fue sustituida por la Tarjeta de Lógica basada en microprocesador, la cual proporcionaba el control de todas las secuencias y tiempos del instrumento, así como el arbitrio y la configuración de los distintos puntos sensados por el instrumento.

El microprocesador también proporcionó el control del reloj digital, la cabeza de impresión térmica, la identificación de puntos, el indicador del número de punto, los niveles de alarma asignados y prácticamente todas las secuencias del instrumento. Así, el nuevo milestone o innovación de ruptura, fueron los microprocesadores de 8 bits.

Registrador Multipunto Digital de $3^{\mathrm{a}} \mathrm{Ge}-$ neración, Speedomax 1650 \& 2500 L\&N ®, (Leeds and Northrup Co. 1992).

Estos instrumentos electró nicos fueron lanzados al mercado a principios de la década de los noventas y ejecutaban prácticamente todas las funciones de los registradores conocidos hasta entonces. También incluí an algunas otras

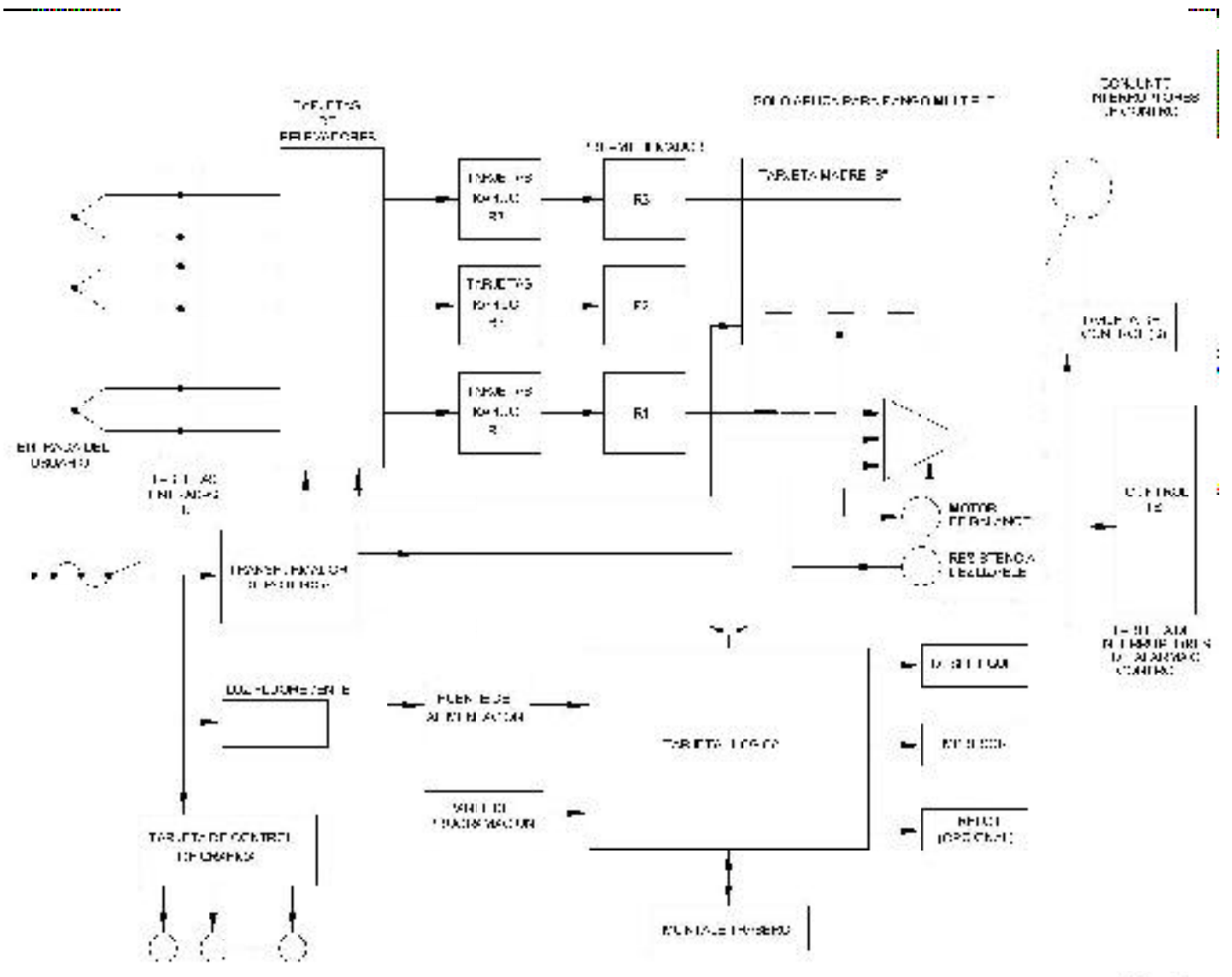

Figura 6. Diagrama de bloques de un registrador multipunto de $2^{a}$ generación 


\section{L.R. Vega-González}

opciones y facilidades adicionales, por ejemplo, el uso del reloj en tiempo real en los registros y su velocidad de gráfica programable. De hecho, prácticamente todas las $\mathrm{f}$ unciones del equipo se hicieron programables y se realizaba un manejo intensivo de alarmas.

Para 1990, los dispositivos electrónicos disponibles eran de muy alta escala de integración, (VLSI), por lo que el milestone de estos registradores fue el uso de una microcomputadora más poderosa, basada en un microprocesador de 16 bits de propósito específico. Este dispositivo permitió el uso de un despliegue digital doble, en el que se podía observar el número del punto medido y el valor de la medición en unidades de ingeniería. También se utilizó un amplificador con control de ganancia variable y convertidores analógico/ digital y digital/analógico de alta exactitud y resolución (Figura 7).

Este instrumento llegó a considerarse como realmente universal, ya que la configuración de todos sus puntos, rangos y niveles de alarma, podían realizarse a través de un módulo programador, interno o externo al instrumento.

En el registrador universal, fue posible programar los rangos de todas sus entradas, dado que el instrumento puede reconocer a través de su memoria EEPROM todos los tipos de termopar (TC), de bulbos de resistencia (RTD), transductores con salida de corriente de 4-20 mA CD o voltajes en los rangos de 1 a $5 \mathrm{~V}$ o hasta $100 \mathrm{~V}$ por medio de divisor de voltaje, con una diferencia entre el valor mayor y el menor (span) mínimo de $1 \mathrm{mV}$ CD.



Figura 7. Diagrama de bloques del registrador multipunto digital de $3^{a}$ generación 
En estos instrumentos vigentes y disponibles en el mercado hasta el día de hoy, la medición analógica se realiza por medio de un amplificador con control de ganancia variable y convertidores analógico/digital y digital/analógico.

En su operación normal, el equipo "reconoce" por programación qué tipo de entrada mide, y asigna la ganancia correspondiente al amplificador, el cual a su vez, impulsa el sistema analógico de balance.

$\mathrm{Al}$ igual que en los registradores de $1^{\mathrm{a}}$ y $2^{\mathrm{a}}$ generació $n$, el sistema de balance está formado por el servoamplificador, el motor de balance y la resistencia deslizable de realimentación. La configuración del instrumento es guiada por programa y se realiza a través de un módulo programador interno con diodos emisores de luz, que guían al usuario indicando los valores que hay que darle al instrumento vía teclado en el recorrido, hacia los distintos pasos de la configuración. Todas sus rutinas están bien establecidas y probadas.

\section{Registrador Multipunto Digital de $4^{\mathrm{a}}$ Generación, Spdx 25000 L\&N ${ }^{\circ}$, (Leeds and Northrup Co. 1995b).}

En la figura 8 se muestra el registrador multipunto digital de $4^{a}$ generación, el cual apareció en el mercado alrededor del año 1995 a finales del siglo pasado. Sus milestones tecnológicos fueron dos innovaciones de ruptura:
- Una computadora de propósito específico, basada en un microprocesador de 32 bits, con multiprocesamiento multitarea asíncrono.

- Despliegue digital tipo plasma.

Otros dispositivos electrónicos integrados, fueron un amplificador mejorado con control de ganancia variable y convertidores analógico/ digital y digital/analógico de alta exactitud y resolución. Asimismo, hace uso de memoria de acceso aleatorio dinámica DRAM.

Este equipo es parte de la categoría de instrumentos universales, constituyendo un verdadero Sistema de Adquisición de Datos, ya que acepta hasta 135 entradas/salidas analógicas, digitales o de pulsos configurables a través de programa en pantalla de plasma. También, incluye impresión multicolor y gráfica plegable.

Pueden configurarse funciones matemáticas y lógicas para un máximo de 60 puntos calculados o pseudopuntos y 50 constantes en memoria para realizar cálculos con los valores de las 135 entradas/salidas en tiempo real.

Algunos ejemplos de pseudopuntos son: el promedio de la señal, promedio del grupo, totalización, flujo másico, detector de picos, entre muchos otros.

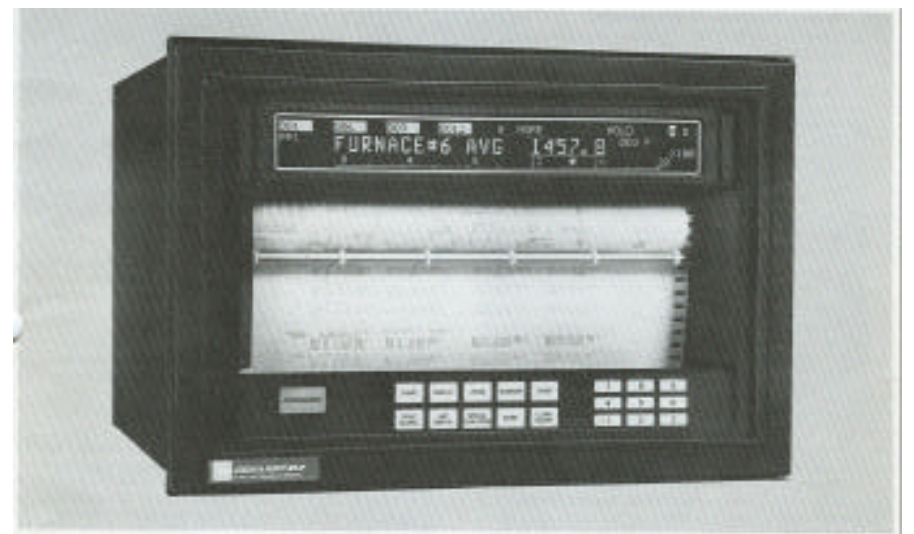

Figura 8. Registrador multipunto digital de $4^{a}$ generación 


\section{L.R. Vega-González}

La velocidad de gráfica puede seleccionarse desde $1 \mathrm{a} 1200 \mathrm{~cm} / \mathrm{hr}$, en intervalos de $1 \mathrm{~cm} / \mathrm{hr}$ y el tiempo por punto se adapta automáticamente a la velocidad de gráfica, para evitar congestión y aglomeramiento de información en la misma.

La configuración puede hacerse vía un teclado para PC AT o XT, o bien, vía un teclado interno al instrumento tipo calculadora.

La estrategia de configuración es la de definir grupos de puntos, de esta manera, el operador puede controlar en forma independiente la operación del despliegue digital, de la operación del sistema de impresión.

Lo que esto significa es que se puede imprimir un grupo de datos, mientras se monitorea otro. Esta función es muy útil en arranques y paros de planta.

La brillantez del indicador tipo plasma, permite una fácil lectura de los puntos y los valores registrados a distancia en el tablero de control. Debido al gran tamaño del indicador, la escala es configurable y se puede asignar a cada uno de los grupos en forma individual. También se cuenta con la función de Zoom para facilitar la identificación de los puntos al expandir su escala bajo solicitud del operador.

\section{El Videoregistrador; Honneywell, Progeny RSX ${ }^{\circledR}$, (Leeds and Northrup Co. 1995a)}

Fue lanzado al mercado en 1995 y se mantuvo vigente en el mercado hasta el 2002. El milestone tecnológico de este instrumento fue la pantalla de cristal líquido ( $L C D)$, a color total, de alta definición. Este dispositivo era reconocido desde 1994 como una innovación de ruptura por sí mismo, ya que significaría un verdadero cambio en todas las áreas de la industria electrónica, (Ohshita, 1994) .

Se trata de un microcomputador dedicado, que cuenta con manejador de discos flexibles de
3.5 pulgadas y tiene capacidad para hasta seis entradas analógicas universales de 4-20 mA CD y salidas analógicas de retransmisión o control, así como entradas-salidas digitales.

Este instrumento contaba con dos lazos de control modulante PID o encendido-apagado, por lo que en realidad se trataba de un videoregistrador-controlador con capacidad de variables calculadas y totalizadores.

Los antecedentes de la combinación registradores-control tiene su antecedente desde los 60 's, donde se montaban en un mismo gabinete y el slidewire se alambraba al controlador como señal de proceso.

\section{Análisis de datos y discusión sobre fabricantes y usuarios de registradores industriales}

En la figura 9, se presenta una línea de tiempo que resume los hallazgos sobre los instrumentos analizados. El período de análisis cubierto es de poco más de un siglo. En la parte superior se indica el nombre del instrumento, mientras que en la parte inferior se señalan los dispositivos innovadores o "milestones" más importantes para el desarrollo.

Puede verse que la difusión de los instrumentos se intensificó a partir de los años sesenta con el advenimiento de los transistores y de los semiconductores, por lo que se cumplen los supuestos de difusión tecnológica de Lissoni \& Metcalfe (1996), que suponen que la adopción de nueva tecnología es más o menos instantánea y que no existen atrasos en la difusión, debidos a lentitud en la imitación, ni influyen aspectos espaciales ni tampoco geográficos.

En estudios hechos a diferentes empresas, Utterback (1986), detectó que la innovación corporativa puede percibirse en diferentes enfoques, el del fabricante y el del usuario. En la industria de la instrumentación, la opinión de los usuarios se toma en cuenta para identificar cuáles son los aspectos o facilidades que deben 


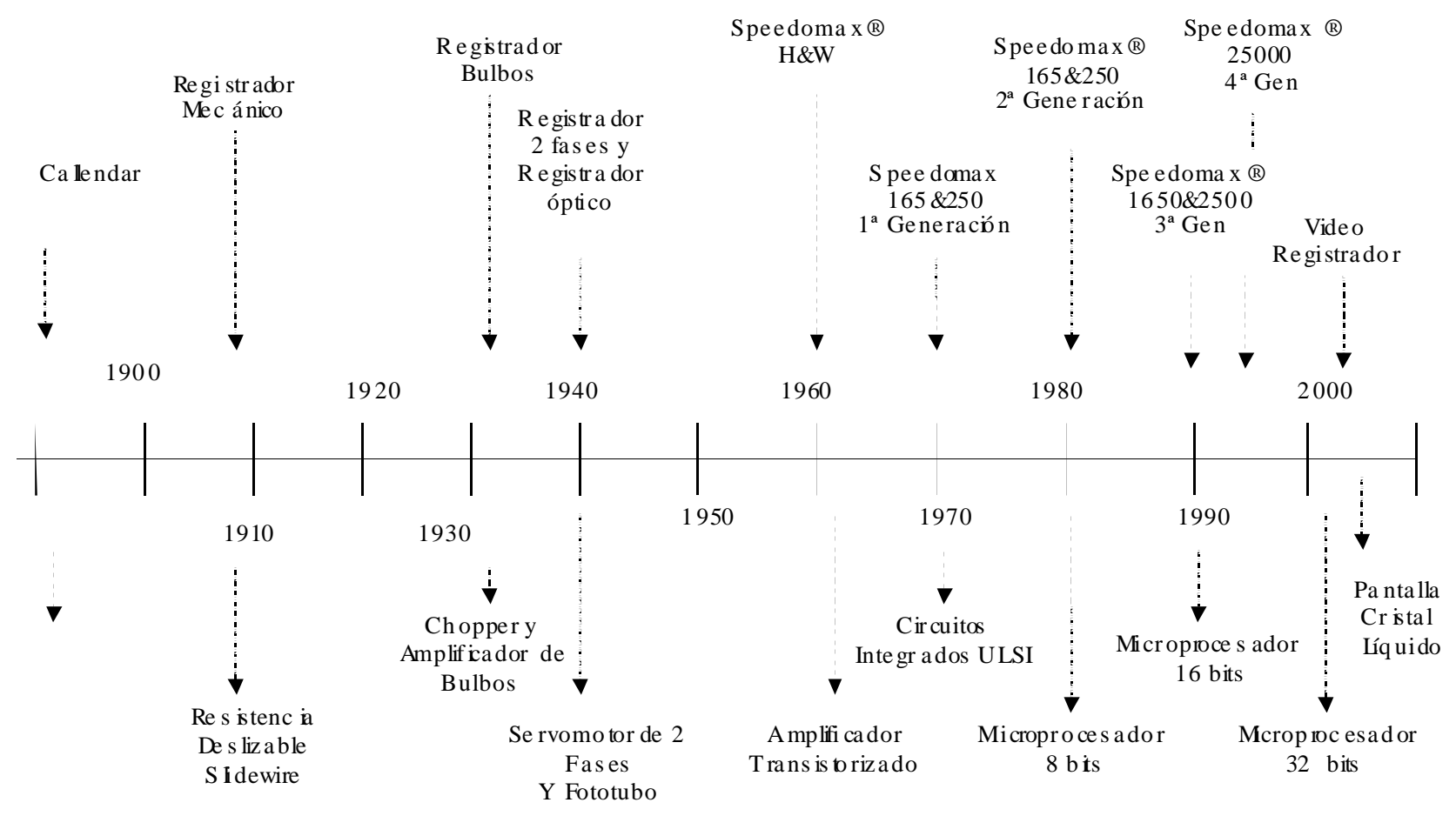

Figura 9. Línea de tiempo de la evolución tecnológica de los registradores industriales

incorporarse a los instrumentos. De esta manera, los proyectos de nuevos productos de las empresas fabricantes, parten de los resultados de entrevistas hechas a los usuarios, quienes, según Von Piel (1988), son en buena medida, una de las fuentes más importantes de la innovación en este campo.

Hoy en día, existe una batalla por el dominio del mercado que libran las empresas fabricantes, en el fondo la pelea real es, entre las tecnologías prevalecientes y las nuevas tecnologías (Nail \& Ahlstrom, 2003). Lo que se busca es la emergencia del diseño tecnológico dominante, aunque se sabe que la tecnología ganadora no necesariamente es la superior en términos de rentabilidad (Arthur, 1996). Entonces, mientras los productos y subsistemas despliegan un proceso de evolución, revolución, maduración, destrucción y reemplazo, el sistema en su conjunto muestra una persistencia remarcable, (Christensen, 1997), esto coincide con la permanencia en el mercado de los registradores de la marca Speedomax por más de sesenta años. La línea de registradores industriales de L\&N, fue y ha sido hasta la fecha muy exitosa por más de seis décadas, aunque la empresa haya perdido la batalla a los embates de la globalización al ser absorbida por la empresa Honeywell a principio de 1990.

Por otra parte, vale la pena señalar que para mantenerse a la vanguardia, las empresas usuarias de instrumentos industriales deben basar su estrategia tecnológica en el desarrollo de competencias; en el sentido propuesto por Campbell \& Sommers, (1997). Esto lo hacen las empresas usuarias, a través de la continua capacitación de los operadores y supervisores en la operación y mantenimiento de los equipos. Este es uno de los principales desafíos, ya que el permanente cambio tecnológico acorta cada vez más el ciclo de vida de los dispositivos.

\section{Conclusiones}

En la figura 9, se observa que a lo largo de la evolución de estos dispositivos industriales, en todos los casos de lanzamiento de nuevos 


\section{L.R. Vega-González}

instrumentos, se disponía de al menos un milestone tecnológico, por lo que se concluye la aparente validez de la hipótesis propuesta al inicio de este trabajo. Sin embargo, es notorio que en muchos otros casos se utilizaron varios dispositivos innovadores en el instrumento bajo estudio. Esto concuerda plenamente con lo propuesto por Iansaty \& West (1997), quienes indican que desde la última década del siglo pasado, las empresas fabricantes de dispositivos tecnológicos utilizan el procedimiento de integración tecnológica (Technology Integration Approach) para refinar y poner a punto las tecnologías utilizadas en cada nuevo producto. El desarrollo de un nuevo producto parte de la plataforma tecnológica previa, a la cual se integran las innovaciones incrementales o radicales disponibles en el momento. Un aspecto relevante y mandatario es, que todos los dispositivos tecnológicos deben trabajar bien y en conjunto, por lo que el proceso de integración inicia con la planeación de un proyecto de investigación y desarrollo (I\&D), la cual provee la ruta de diseño, ingeniería y manufactura.

Otra conclusión es que en el caso de los registradores industriales se cumplen plenamente las características de innovación principales de la electrónica de consumo, propuestas por Cawson (1996); es decir, la innovación en la instrumentación de indicación, registro y adquisición de datos se da en base a estándares, propiciada por la tecnología digital, con lo que se generan familias de productos en lugar de productos discretos. Esto es evidente con la familia Speedomax de L\&N analizada. El fenómeno también existe y es convergente con lo que sucede en las industrias relacionadas como la computación, las telecomunicaciones y la electró nica profesional.

Finalmente, dado que los analistas vislumbran la aparición de nuevos dispositivos ópticos de efecto cuántico que transformarán las industrias de la electrónica, del cómputo y de las comunicaciones (Smith \& Antoniadis, 1990); es previsible que los fabricantes de registradores industriales en el futuro cercano re- tomen, mejoren y optimicen las tecnologías propuestas desde 1940 por la empresa Tagliablue Manufacturing Co; para desarrollar instrumentos de registro y adquisición de datos a partir de sensores ópticos y transmisores inalámbricos.

\section{Agradecimientos}

Agradezco al Sr. Joe Kulak, Director de Marketing Internacional de Leeds \& Northrup Company, por su autorización para publicar la información técnica sobre los instrumentos de su firma. También expreso mi profundo agradecimiento al Tec. Pedro Hernández Segovia por su ayuda en la realización de los dibujos de este trabajo.

\section{Referencias}

[1] Administración de proyectos, http://www.faccat.com.br/dti/pds.htm

[2] Cromatography Milestones, http://www.lcgcelectronic.com/050504/P age_8.asp

[3] IEEE History Center, http://www.ieee.org/organizations/histo ry_center/milestonesArthur B. (1996). Increasing Returns and New World of Business. Harvard Business Review, No. 74, pp.100-109.

[4] Pediatría, http://www.mcghealth.org/Greystone/sa dult/pedi at rics/speech.html

[5] Solar Track, http://www.tapthesun.com/HTML/Deve lopment\%20Milestones /

Campbell \& Sommers (1997). Estrategia basada en competencias críticas.

Cawson A. (1996). Innovation and Consumer Electronics; in: The handbook of Industrial Innovation, Dodgson Mark, Rothwell Roy, Editors; Edward Elgar Publishing Ltd, Uk, pp. 145-153.

Clark W.R. (1966). Recorders. Reprint From Encyclopaedia of Indus trial Chem ical Analysis, Vol. 3, John Wiley \& Sons, Inc., EUA.

Christensen C.M. (1997). The Innovator's Dilemma: When new Tech nol ogies Cause 
DOI: http://dx.doi.org/10.22201/fi.25940732e.2007.08n1.002

Relación entre algunos milestones tecnológicos y la evolución de los registradores industriales

Great Firms to fall. Harvard Business School Press, Boston Mass. EUA.

Iansati M. and West J. (1997). Technology Integration. Harvard Business Review, May-June, pp. 69-78.

Leeds \& Northrup Co. (1971). Catálogo general de instrumentación industrial, No. HO.0001 171, Philadelphia Pennsylvania, EUA.

Leeds \& Northrup Co. (1972). Catálogo general de instrumentación industrial, No. HO.0001-INT 172, Phil a del phia Penn sylvania, EUA.

Leeds \& Northrup Co. (1975). Speedomax HEW, y amplificadores de estado sólido, Manual de instrucciones 077990 Ed. 17 y suplementos 077990/177232. Philadel phia Penn sylvania, EUA.

Leeds \& Northrup Co. (1979). Catálogo general de instrumentación industrial No. HO.0001 1179, Philadelphia Pennsylvania, EUA.

Leeds \& Northrup Co. (1980) Registradores industriales Speedomax 165E250, Manual de operación y servicio 177976 Rev. B, Phila del phia Penn sylvania, EUA.

Leeds \& Northrup Co. (1992), Registradores Multipunto Speedomax 1650\&2500, Hoja de Datos CO.7111-DS, Phil a del phia Penn sylvania, EUA.

Leeds \& Northrup Co. (1995a). The Progeny Family. Boletín PO.0011BR-7/94, Phil adel phia Penn sylvania, EUA.
Leeds \& Northrup Co. (1995b). Registrador Multipunto Speedomax 25000, Hoja de Datos CO.7130-DS, Philadelphia Penn sylvania, EUA.

Lissoni F. and Metcalfe J.S. (1996). Diffusion of Innovation Ancient and Modern: a Review of the Main Themes; in: The handbook of industrial innovation, Dodgson Mark, Rothwell Roy, Editors; Edward Elgar Publishing Ltd, Uk, pp. 106-144.

Merriam Webster's Collegiate Dictionary (1996). 10th. Edition, Springfield Massachu setts USA.

Nair A and Ahlstrom D. (2003). Delayed Creative Destruction and the Coexistence of Technologies. Journal of Engineering and Technology Management, Vol. 20, pp. 345-365. Elsevier, Science Direct.

Ohshita-Shinji (1994). Liquid Cristal Display (LCD) and Future Applied Products. Science $\mathcal{E}$ Technology in Japan, No. 51, pp. 43-45.

Smith H.I. and Antoniadis D. (1990). Fabri cating Quantum Effect Devices: in: Seeking a Radically new electronics, Technology Review. April, pp. 26-40.

Utterback J. (1986). Innovation and Corpo rate Strategy. International Journal of Technology Management, Vol. 1. Nos.1/2, pp.119-132.

Von Hippel E. (1988). The Sources of Inno vation. Oxford Univer sity Press, New York, Oxford.

\section{Semblanza del autor}

Luis Roberto Vega-González. Es ingeniero mecánico electricista por la Facultad de Ingeniería de la UNAM. Obtuvo la maestría en ingeniería de sistemas en el área de planeación por la DEPFI-UNAM y la maestría en administración de las organizaciones por la División de Estudios de Posgrado de la Facultad de Contaduría y Administración de la UNAM. Asimismo, se especializó en gestión de la innovación tecnológica por el Centro para la Innovación Tecnológica y la FCyA de la UNAM. Durante más de veinte años, colaboró en diversas firmas nacionales e internacionales con proyectos en las áreas de instrumentación, control y potencia. Actualmente es coordinador de vinculación y gestión tecnológica del Centro de Ciencias Aplicadas y Desarrollo Tecnológico (CCADET) de la UNAM. Ha conducido negociaciones, convenios, contratos y realizado diferentes actividades de gestión tecnológica para cerca de dos centenares de proyectos en diversas entidades de la UNAM. 